\title{
Detection of recurrent membranous nephropathy in a renal allograft by biomarker
}

\begin{abstract}
Primary membranous nephropathy (MN) is an autoimmune kidney disease, mediated by IgG4 to phospholipase A2 receptor (PLA2R) expressed on podocytes. Primary MN recurs in renal allografts. Using biological markers of primary MN (PLA2R and IgG4), we identify a case of recurrent primary $\mathrm{MN}$ in a renal allograft.
\end{abstract}

Keywords: nephropathy, autoimmune kidney disease, glomerulopathy, HIV
Volume 3 Issue 3 - 2018

\author{
Xu Zeng, Karl L Womer, Michael J Casey, \\ Alfonso H Santos, Kawther Alquadan, \\ William Clapp \\ University of Florida, USA
}

Correspondence: Xu Zeng, 4800 SW 35th Drive, UF Health Pathology Laboratories, Gainesville, Florida-2608, Fax 352-265990I, Tel 352-265-0680, Ext 72028, Email xu.zeng@ufl.edu

Received: March 26, 2018 | Published: June 21, 2018

\section{Introduction}

Primary membranous nephropathy (MN) is an immune complex mediated glomerulopathy characterized by subepithelial immunoglobulin $\mathrm{G}$ ( $\operatorname{IgG}$ ) deposits along the glomerular basement membrane. ${ }^{1}$ Recent research reveals that primary MN is mediated by an autoantibody to the phospholipase A2 receptor (PLA2R) expressed solely on podocytes. ${ }^{2}$ The majority of autoantibody in the subepithelial deposits belongs to the IgG4 subclass. ${ }^{3}$ Primary MN manifests generally as a slowly progressive kidney disease that follows "the rule of one thirds", that is spontaneous remission in one third, persistent proteinuria in another one third, and more progressive course in the remaining third. ${ }^{4,5}$ The last group of patients often progresses from glomerulopathy to end-stage renal disease in 10 years. Like any other end-stage renal disease, management for this group of patients relies on renal replacement therapy with either dialysis or renal transplantation. However, primary MN can recur in renal allografts, ${ }^{6}$ so that the differential diagnosis of membranous nephropathy in the renal allograft always includes recurrence of primary MN versus de novo MN. ${ }^{7}$ Herein, we identify a case of recurrent primary MN in a renal allograft by applying biological markers of IgG4 and PLA2R, which commonly apply to primary MN.

\section{Case description}

A 69 year-old male came to kidney transplant clinic for evaluation of foamy urine. Significant history included end-stage renal disease due to membranous nephropathy. He received a deceased donor kidney transplant in 2015 from a 48 year-old African American female donor. Alemtuzumab was used for induction. Other clinical history included hypertension, gastro-esophageal reflux disease and posttransplant erythrocytosis. Medications included carvedilol, cinacalcet, clonidine, mycophenolate mofetil, prednisone, and tacrolimus. Physical examination was unremarkable. No edema was seen in the extremities. Labs showed stable renal function at baseline with serum creatinine of $1.56 \mathrm{mg} / \mathrm{dL}$. His tacrolimus was within the therapeutic range. Urinalysis was remarkable for new onset +2 proteinuria (Table 1). Serology for hepatitis B and hepatitis C were negative. HIV was negative. Serum protein electrophoresis was within normal range. Because of prior history of membranous nephropathy in the native kidney, there was a concern for recurrent disease in the transplant kidney.
Table I Pertinent labs

\begin{tabular}{|c|c|}
\hline FK506 & $7.2 \mathrm{ug} / \mathrm{L}$ \\
\hline Sodium & $143 \mathrm{mEq} / \mathrm{L}$ \\
\hline Potassium, Serum & $4.5 \mathrm{mEq} / \mathrm{L}$ \\
\hline Chloride, Serum & $106 \mathrm{mEq} / \mathrm{L}$ \\
\hline Urea Nitrogen & $20 \mathrm{mg} \%$ \\
\hline Creatinine & 1.56 mg\% \\
\hline Estimated GFR & 52 \\
\hline Glucose, Serum & 101 mg\% \\
\hline Calcium, Serum & $8.9 \mathrm{mg} \%$ \\
\hline \multicolumn{2}{|l|}{ Urine analysis } \\
\hline Color & Yellow \\
\hline Clarity & Clear \\
\hline Specific Gravity & 1.014 \\
\hline $\mathrm{Ph}$ & 6.5 \\
\hline Protein & $2+$ \\
\hline Glucose & Negative \\
\hline Ketones & Negative \\
\hline Bilirubin & Negative \\
\hline Nitrite & Negative \\
\hline Urobilinogen & 0.2 \\
\hline Leukocytes & Negative \\
\hline RBC & $0-2$ \\
\hline WBC & $0-5$ \\
\hline Epithelial Cells & None seen \\
\hline Bacteria & None seen \\
\hline
\end{tabular}

A biopsy of the allograft kidney was performed. Light microscopy revealed renal cortical tissue with mild patchy tubular atrophy and interstitial fibrosis. Interstitial inflammation was minimal without features of acute cellular or antibody mediated rejection. No 
morphologic features of acute or chronic toxicity of tacrolimus were seen. The glomeruli showed normal mesangial cellularity. Three out of 35 glomeruli (8.5\%) were globally sclerotic. No segmental sclerosis in the glomerular tuft was identified. The thickness of glomerular capillary loops was within normal range (Figure 1A). No prominent "spike" was identified. Immunofluorescence showed diffuse, granular glomerular capillary loops staining for $\operatorname{IgG}(3+)$ and $\mathrm{C} 3$ $(3+)$ (Figure 1B). Under electron microscopy, scattered subepithelial electron-dense deposits were identified without glomerular basement membrane reaction. Podocyte foot processes were diffusely effaced (Figure 1C) (Figure 1D). Based on the foregoing findings, a diagnosis of "membranous nephropathy, stage I" was established.

Subsequently, markers for primary MN were applied to the renal allograft biopsy. Immunofluorescence for IgG subclass (IgG1, $\operatorname{IgG} 2$, IgG3 and IgG4) was performed and it showed granular staining along the glomerular capillary loops for IgG1 (1-2+), IgG3 $(2+)$ and IgG4 (3+). Staining for IgG2 was negative. Thus IgG4 was the predominant $\mathrm{IgG}$ in the subepithelial deposits (Figure 1E). By immunohistochemistry, immune deposits stained positive for Phospholipase A2 receptor (Figure 1F). These findings indicated a recurrent membranous nephropathy in the renal allograft.

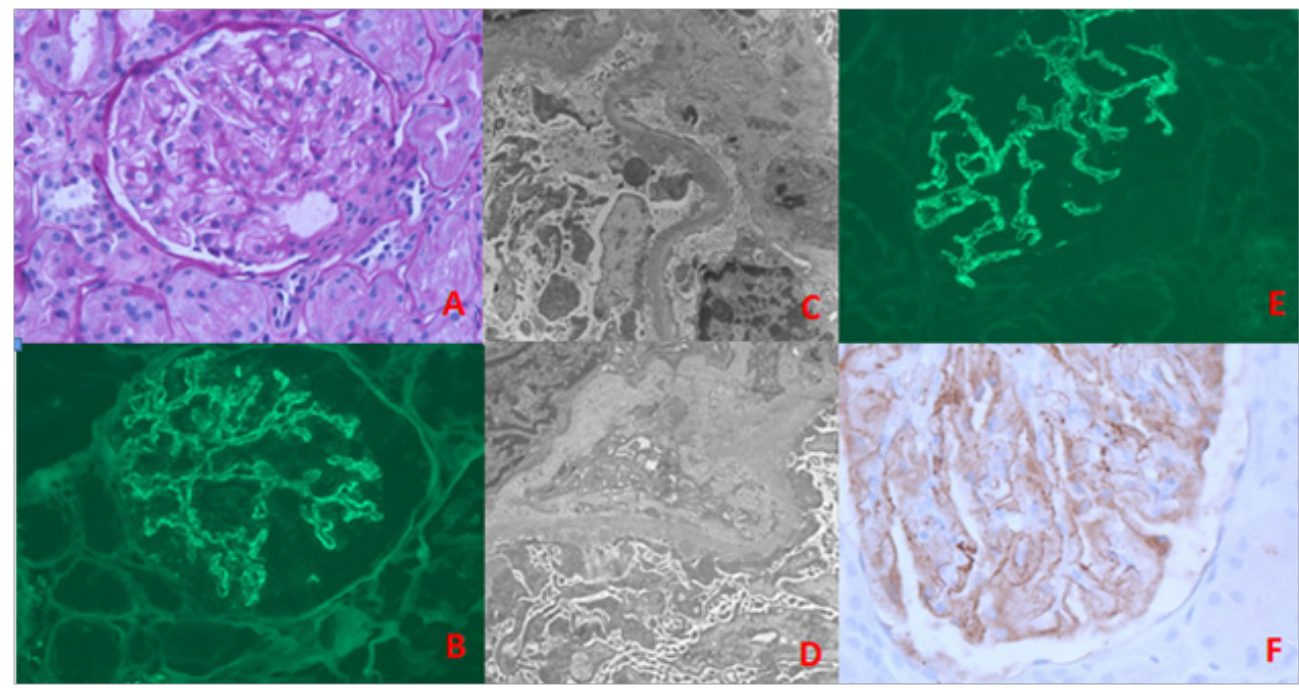

Figure I Renal biopsy findings: (A) Light Microscopy showing normal mesangial cellularity without prominent thickened glomerular capillary loops (PAS stain) (B) Immunofluorescence showing scatter granular glomerular capillary loops staining for IgG. (C, D) Electron microscopy demonstrating scattered subepithelial electron dense immune deposits without glomerular basement membrane reaction (stage I). The podocyte foot processes are effaced. (E) Immunofluorescence showing immune deposits along glomerular capillary loops staining for lgG4. (F) Immunohistochemistry showing immune deposits along glomerular capillary loops also staining for PLA2R.

\section{Discussion}

The differential diagnosis for patients presenting with proteinuria after kidney transplant is broad, including de novo or recurrent glomerular disease. Common entities include focal and segmental glomerulosclerosis, chronic transplant glomerulopathy, minimal change disease secondary to medication, and rarely membranous nephropathy. In the present patient, because of history of membranous nephropathy in the native kidneys (although we are unable to retrieve the native kidney biopsy report or slides to confirm the prior diagnosis in native kidney), new onset proteinuria strongly suggests the possibility of recurrent primary MN. Biopsy of the allograft kidney was necessary for the early detection and treatment of glomerular disease in this patient. However, diagnosis of early, stage I membranous nephropathy by light microscopy alone is quite a challenge in this case. The glomerulus exhibits no apparent abnormalities under light microscopy (Figure 1A). The glomerular basement membrane usually is still thin and delicate, and no prominent thickening or "spike" formation can be seen (Figure 1A). Combination of light microscopy, immunofluorescence and electron microscopy are very helpful to detect early stage MN. Immunofluorescence study is more sensitive in detecting immune deposits than light microscopy. Classic immunofluorescence findings in MN are global IgG and C3 deposits along the glomerular basement membrane (Figure 1E). Electron microscopy is able to further identify and confirm the location of subepithelial immune complexes (Figure 1C) (Figure 1D), which are a major criteria to establish the diagnosis of $\mathrm{MN} .{ }^{1} \mathrm{~A}$ diagnosis of $\mathrm{MN}$ in the renal allograft always raises the suspicion for either recurrent or de novo MN. About $30 \%$ of primary MN recur in renal allografts, ${ }^{8}$ which is seen most commonly 2 to 3 years post transplantation. ${ }^{9}$ The PLA2R, a marker for primary MN, has been shown to be a useful tool to distinguish between recurrent and de novo $\mathrm{MN}$ in renal allografts, ${ }^{10}$ with a sensitivity of $83 \%$ and specificity of $92 \%$ in recurrent MN. ${ }^{11}$ To the best of our knowledge, using IgG4 staining to identify recurrent $\mathrm{MN}$ in renal allograft has not been reported. Subclasses of IgG include $\operatorname{IgG} 1, \mathrm{IgG} 2, \operatorname{IgG} 3$ and $\operatorname{IgG} 4$, in which IgG1 is the dominant circulating IgG $(66 \%)$ while $\operatorname{IgG} 4$ comprises only $4 \% \cdot{ }^{12} \operatorname{IgG} 4$ has weak disulfide bonds between two heavy chains, so IgG4 is able to dissociate into two half IgG4 molecules, each having a single light and heavy chain, that are able to reassemble with the other half IgG4 molecule to form hetero-bivalent IgG4 with two different specificities. ${ }^{13}$ Therefore, bispecific IgG4 is specific to cross-linking of antigens and serve to down-regulate the immune response. ${ }^{12,13}$ In primary MN, the IgG4 is the predominant IgG subclass in the subepithelial deposits and could be responsible for protection through cross-linking of antigen. ${ }^{3,14}$ Of note, IgG4 is the predominant, but not the only IgG subclass in the 
subepithelial deposits in primary MN. Other IgG subclasses, including $\operatorname{IgG} 1, \operatorname{IgG} 2$ and $\operatorname{IgG} 3$ are also present in small varying amount. ${ }^{15}$ However, only demonstration of dominant IgG4 staining is significant for the diagnosis of primary MN. ${ }^{16}$ In contrast, while IgG1 subclass is dominant in $\mathrm{MN}$ secondary to malignancy, ${ }^{15}$ and more important de novo $\mathrm{MN}$ in renal allograft. ${ }^{13}$ Thus, in practice, it is better to include the entire IgG subclass to examine for primary MN. In present case, dominant staining for IgG4 but not IgG1 subclass is very helpful to identify recurrent $\mathrm{MN}$ in the renal allograft.

\section{Conclusion}

In conclusion, biomarkers of PLA2R and IgG4 for primary MN are useful to detection of recurrent $\mathrm{MN}$ in renal allografts and should be included in routine pathology practice, although the sensitivity and specificity for IgG4 to detect recurrent $\mathrm{MN}$ have not been reported.

\section{Acknowledgements}

None.

\section{Conflict of interest}

The author declares there is no conflict of interest.

\section{References}

1. Glassock RJ. The pathogenesis of idiopathic membranous nephropathy: a 50-year odyssey. Am J Kidney Dis. 2010;56(1):157-67.

2. Beck LH, Bonegio RG, Lambeau G, et al. M-type phospholipase A2 receptor as target antigen in idiopathic membranous nephropathy. $N$ Engl J Med. 2009;361(1):11-21.

3. Doi T, Mayumi M, Kanatsu K, et al. Distribution of IgG subclasses in membranous nephropathy. Clin Exp Immunol. 1984;58(1):57-62.

4. Monga G, Mazzucco G, Di Belgiojoso GB, et al. The presence and possible role of monocyte infiltration in human chronic proliferative glomerulonephritides. Light microscopic, immunofluorescence, and histochemical correlations. Am J Pathol. 1979;94(2):271-284.
5. Yang N, Isbel NM, Nikolic-Paterson DJ, et al. Local macrophage proliferation in human glomerulonephritis. Kidney Int. 1998; 54(1):14351.

6. Ponticelli C, Glassock RJ. Posttransplant recurrence of primary glomerulonephritis. Clin J Am Soc Nephrol. 2010;5(12):2363-72.

7. Abbas F, El Kossi M, Jin JK, et al. De novo glomerular diseases after renal transplantation: How is it different from recurrent glomerular diseases? World J Transplant. 2017;7(6):285-300.

8. Josephson MA, Spargo B, Hollandsworth D, et al. The recurrence of recurrent membranous glomerulopathy in a renal transplant recipient: case report and literature review. Am J Kidney Dis. 1994;24(5):873-8.

9. Debiec H, Martin L, Jouanneau C, et al. Autoantibodies specific for the phospholipase A2 receptor in recurrent and De Novo membranous nephropathy. American J of Transplantation. 2011;11:2144-2152.

10. Larsen CP, Walker PD. Phospholipase A2 receptor (PLA2R) staining is useful in the determination of de novo versus recurrent membranous glomerulopathy. Transplantation. 2013;95(10):1259-62.

11. Sigal LH. Basic science for the clinician 58: IgG subclasses. J Clin Rheumatol. 2012;18(6):316-8.

12. Aalberse RC, Schuurman J. IgG4 breaking the rules. Immunology. 2002;105(1):9-19.

13. Noël LH, Aucouturier P, Monteiro RC, et al. Glomerular and serum immunoglobulin $\mathrm{G}$ subclasses in membranous nephropathy and antiglomerular basement membrane nephritis. Clin Immunol Immunopathol. 1988;46(2):186-94.

14. Ohtani H, Wakui H, Komatsuda A, et al. Distribution of glomerular IgG subclass deposits in malignancy-associated membranous nephropathy. Nephrol Dial Transplant. 2004;19(3):574-9.

15. Imai $\mathrm{H}$, Hamai $\mathrm{K}$, Komatsuda $\mathrm{A}$, et al. IgG subclasses in patients with membranoproliferative glomerulonephritis, membranous nephropathy, and lupus nephritis. Kidney Int. 1997;51(1):270-6.

16. Sudesh P, Makker MD, Tramontano A. Idiopathic Membranous Nephropathy: An Autoimmune Disease. Semin Nephrol. 201; 31(4):333340. 